\title{
Effect of two bleaching regimens on enamel bonding performance
}

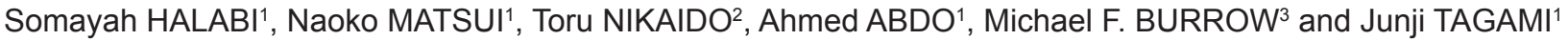 \\ ${ }^{1}$ Cariology and Operative Dentistry Department, Graduate School of Medical and Dental Sciences, Tokyo Medical and Dental University, 1-5-45 \\ Yushima, Bunkyo-ku, Tokyo 113-8510, Japan \\ ${ }^{2}$ Department of Operative Dentistry, Division of Oral Functional Science and Rehabilitation, School of Dentistry, Asahi University, 1851 Hozumi, \\ Mizuho, Gifu 501-0296, Japan \\ ${ }^{3}$ Faculty of Dentistry, University of Hong Kong, Hong Kong, China \\ Corresponding author, Naoko MATSUI; E-mail: matsui.ope@tmd.ac.jp
}

This study evaluated the post-bleaching bonding performance of a one-step and two-step self-etch adhesive (SEA) to bleached enamel using in-office or at-home bleaching. Bovine enamel surfaces were treated with an in-office or at-home bleaching agent and divided into four post-bleach time periods: immediate, one day (1d), one week (1w) and one month (1m) before application of SEA systems. Enamel was bonded using the following procedures: Group 1: one-step SEA application (BeautiBond Multi, BBM), Group 2: two-step SEA application (FL-Bond II, FLBII). Microshear bond strengths ( $\mu$ SBS) were measured 24-h after bonding for each of the postbleaching time periods. Acid-base resistant zones (ABRZs) were observed under SEM. Data were analyzed using three-way ANOVA. Bleaching caused a significant decrease of the immediate $\mu$ SBSs for both adhesives $(p \leq 0.005)$ then increased. Thick ABRZs were observed in BBM and FLBII after bleaching. Enamel $\mu$ SBSs were adversely affected by bleaching. Thick ABRZ was created with bleaching.

Keywords: Bleaching, Enamel, Bonding performance, Microshear bond strength, Acid-base resistance zone

\section{INTRODUCTION}

Bleaching with carbamide peroxide or hydrogen peroxide is a safe, evidence-based and conservative alternative to more invasive esthetic treatments, such as the placement of veneers or full crowns on discolored teeth.

Different regimens for bleaching teeth are available (at-home or in-office). These regimens use various concentrations of bleaching agents. In general, in-office bleaching is done with high concentrations of hydrogen peroxide (25-35\%), whilst at-home bleaching products, with a few exceptions, contain carbamide peroxide in varying concentrations, ranging from 5 to $30 \%^{11}$. The in-office technique has emerged as more popular than home use because highly concentrated products usually provide a faster tooth whitening outcome (the higher the concentration of bleaching solution, the more quickly a shade change will occur) ${ }^{2}$.

One of the adverse effects of tooth bleaching has been the reported decrease in bond strengths for resin-based materials to enamel immediately after the bleaching procedure. The reduction in bond strength has been ascribed to the oxygen released from bleaching agents into tooth structure that inhibits resin polymerization ${ }^{3}$.

According to the clinical application steps, selfetch adhesive (SEA) systems have been classified as one-step or two-step adhesives. One-step SEA systems, combine acidic functional monomers, hydrophilic and hydrophobic monomers, water and organic solvents in a single solution. However, two-step SEA systems contain

Color figures can be viewed in the online issue, which is available at J-STAGE.

Received Aug 5, 2019: Accepted Dec 5, 2019

doi:10.4012/dmj.2019-239 JOI JST.JSTAGE/dmj/2019-239 an acidic monomer that simultaneously etches and primes the tooth substrate, prior to the application of a separate bonding resin. The acidic functional monomer is believed to play several important roles during the etching and priming steps to form a chemical bond to the calcium of the tooth substrate.

A new version of a fluoride-releasing adhesive system has been introduced in order to inhibit the initiation of recurrent caries at the tooth-restoration interface ${ }^{4,55}$. Some researchers demonstrated significant cariostatic and antibacterial effects of fluoride-releasing restorative materials $^{6,7)}$.

When a resin-based adhesive is bonded to the enamel and dentin, an acid-base resistant zone (ABRZ) is formed at the adhesive-tooth substrate interface. This was first reported by Tsuchiya et al. ${ }^{8}$. The ABRZ is characterized as a structure at the resin-tooth substrate interface which remains after an acid-base challenge. The first report was based on the resin-dentin interface, but recent studies have also demonstrated the formation of ABRZ at enamel interfaces with one-, two- and threestep etch and rinse adhesive systems as well as SEA systems $^{9-12)}$. The ABRZ seems to be associated with sealing of restoration margins and therefore enhancing restoration durability $y^{9,10,13,14)}$. Shinohara et al. reported that the thickness of the ABRZ was increased by fluoride ion release from the adhesive ${ }^{15}$. It has been reported that bleaching agents can affect the morphology of the enamel ABRZ and create thicker ABRZs when an inoffice bleaching system was used ${ }^{8}$. However, the effect of at-home bleaching on ABRZ morphology has yet to be reported. 
Therefore, the present study aimed to evaluate the effect of different bleaching regimens on enamel bonding performance using a one- and two-step SEA systems at various time intervals after completion of the bleaching process and before adhesive application. In-office and athome bleaching materials were used prior to adhesive application and tested by means of a microshear bond strength $(\mu \mathrm{SBS})$ test. Micro-morphological features of the ABRZ were assessed using scanning electron microscopy (SEM).

\section{MATERIALS AND METHODS}

\section{Materials used in this study}

The materials used in this study are described in Table 1. A one-step SEA (BeautiBond Multi, BBM, Shofu Dental, Kyoto, Japan) and a two-step SEA (FL-Bond II, FLBII, Shofu Dental) were used. BBM is a fluoridefree adhesive which contains a carboxylic acid monomer with water and organic solvents in the one bottle adhesive. FLBII is also composed of the same functional carboxylic acid monomer in the self-etching primer and has fluoride-releasing components of F-PRG $r$ and S-PRG fillers. These filler particles were created using pre-reacted-glass-ionomer (PRG) technology that has been previously described ${ }^{16)}$. A resin composite, Beautifil II (Shofu Dental), is a fluoride-containing hybrid resin composite and used as part of manufacture of specimens. An in-office bleaching agent (Hi-Lite office bleaching, Shofu Dental) containing $35 \%$ hydrogen peroxide $(\mathrm{pH}=3.9-4.1)$ and an at-home bleaching agent (Hi-Lite shade up home bleaching, Shofu Dental) containing $10 \%$ carbamide peroxide gel $(\mathrm{pH}=6-7)$ were the bleaching agents selected.

\section{Specimen preparations}

Specimen preparations are illustrated in Fig. 1. One hundred and twenty-six bovine incisors, free from structural cracks and defects, were used in this study. Roots were resected $1 \mathrm{~mm}$ above the cemento-enamel junction using a diamond saw (Isomet 1000, Buehler, Lake Bluff, IL, USA) under water-cooling. Each tooth was sectioned to obtain one fragment from the labial surface per tooth $(10 \times 10 \mathrm{~mm})$. The enamel surfaces were wet-ground with 600-grit silicon carbide abrasive papers to create a flat surface and standardized smear layer ${ }^{3,17)}$, as well as to ensure a flat surface which is parallel to the applied shear stress during the $\mu$ SBS test.

The specimens were divided into three main groups according to bleaching regimen: 1: no bleaching (control), 2: in-office bleaching (Hi-Lite office), and 3: athome bleaching (Hi-Lite shade up). The control group was subdivided into the one-step (BBM) and two-step (FLBII) self-etching adhesive groups. The two bleaching

Table 1 Materials used in this study

\begin{tabular}{|c|c|c|}
\hline Material & Composition & Application procedures \\
\hline $\begin{array}{l}\text { FL-Bond II } \\
\text { (FLBII) }\end{array}$ & $\begin{array}{l}\text { Primer: Carboxylic acid monomer, 6-MHPA, } \\
\text { Water, Solvent, Photo-initiator } \\
\text { Bond: HEMA, UDMA, TEGDMA, S-PRG filler, } \\
\text { Photo-initiator }\end{array}$ & $\begin{array}{l}\text { Apply primer to tooth surface, and leave in place } \\
\text { for } 10 \mathrm{~s} \text {. Dry with air stream. Apply bond and } \\
\text { create a uniform film with gentle air stream. } \\
\text { Light cure for } 10 \mathrm{~s}\end{array}$ \\
\hline $\begin{array}{l}\text { BeautiBond } \\
\text { Multi (BBM) }\end{array}$ & $\begin{array}{l}\text { Bis-GMA, carboxylic acid monomer, TEGDMA, } \\
\text { phosphonic acid-based monomers, acetone, } \\
\text { purified water }\end{array}$ & $\begin{array}{l}\text { Apply using a microbrush. Leave undisturbed for } \\
10 \mathrm{~s} \text { after application. Dry thoroughly for } 3 \mathrm{~s} \text { with } \\
\text { maximum air pressure. Light cure for } 10 \mathrm{~s} \text {. }\end{array}$ \\
\hline Beautifil II & $\begin{array}{l}\text { Filler: } 83.3 \mathrm{wt} \%(68.6 \mathrm{vol} \%) \text { Multi-functional } \\
\text { glass and S-PRG filler based on } \\
\text { fluoroboraluminosilicate glass particle size } \\
\text { range: } 0.01-4.0 \mu \mathrm{m} \text { (mean } 0.8 \mu \mathrm{m}) \\
\text { Base resin: Bis-GMA, TEGDMA }\end{array}$ & $\begin{array}{l}\text { Place into tygon tubes on the enamel surface. } \\
\text { Light cure for } 40 \mathrm{~s} \text {. }\end{array}$ \\
\hline $\begin{array}{l}\text { Hi-Lite } \\
\text { (Office } \\
\text { bleaching) }\end{array}$ & $\begin{array}{l}\text { Powder: Potassium persulphinate, manganese } \\
\text { sulfate, monohydrate, hydrated amorphous } \\
\text { silica, mixed sodium and calcium salt of poly } \\
\text { (MVE/MA), pigment } \\
\text { Liquid: } 35 \% \text { hydrogen peroxide, water }\end{array}$ & $\begin{array}{l}\text { One scoop of powder and three drops of liquid } \\
\text { were mixed for } 30 \mathrm{~s} \text {. Apply the paste in } \\
1-2 \mathrm{~mm} \text { thickness and left on the surface for } \\
6 \text { min following light curing for } 3 \mathrm{~min} \text {, and left for } \\
\text { another } 2 \text { min then wiped off by cotton. Repeated } \\
3 \text { times then washed with water and dried gently. }\end{array}$ \\
\hline $\begin{array}{l}\text { Hi-Lite } \\
\text { shade up } \\
\text { (Home } \\
\text { bleaching) }\end{array}$ & $10 \%$ carbamide peroxide gel & $\begin{array}{l}\text { apply using brush and left for } 2 \text { hours then } \\
\text { washed with water. Repeated on } 14 \text { consecutive } \\
\text { days. Stored in distilled water at } 37^{\circ} \mathrm{C} \text { between } \\
\text { bleaching cycles. }\end{array}$ \\
\hline
\end{tabular}

Manufacturer: Shofu Dental, Kyoto, Japan

Bis-GMA: bisphenol-A-glycidyl methacrylate; HEMA: 2-hydroxyethyl methacrylate; TEGDMA: triethyleneglycol dimethacrylate; UDMA: urethane dimethacrylate 


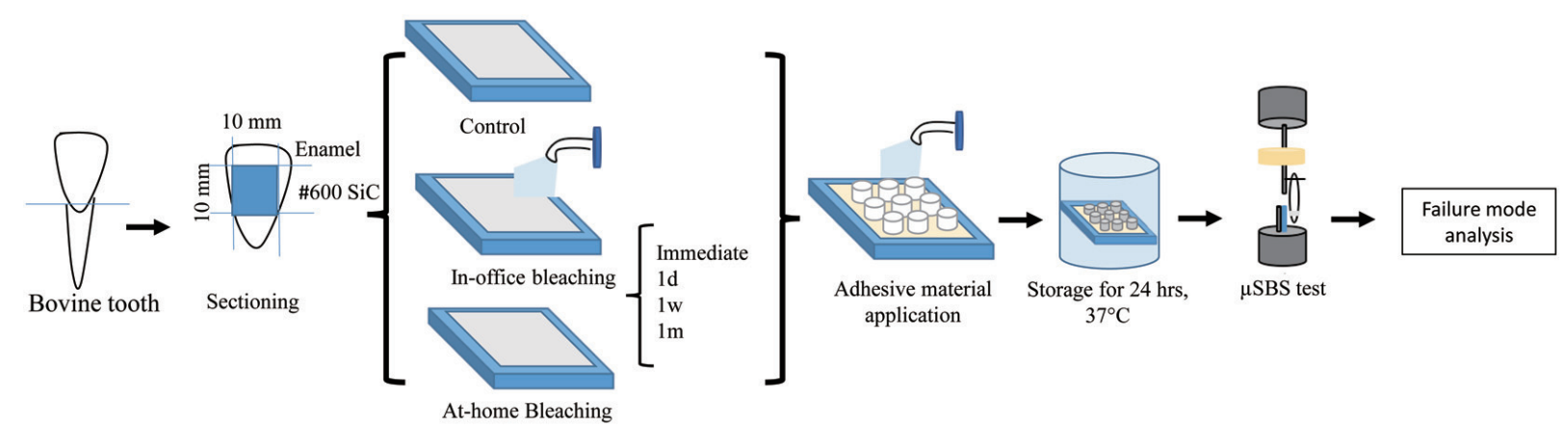

Fig. 1 Methodology for specimen preparations for the $\mu$ SBS test.

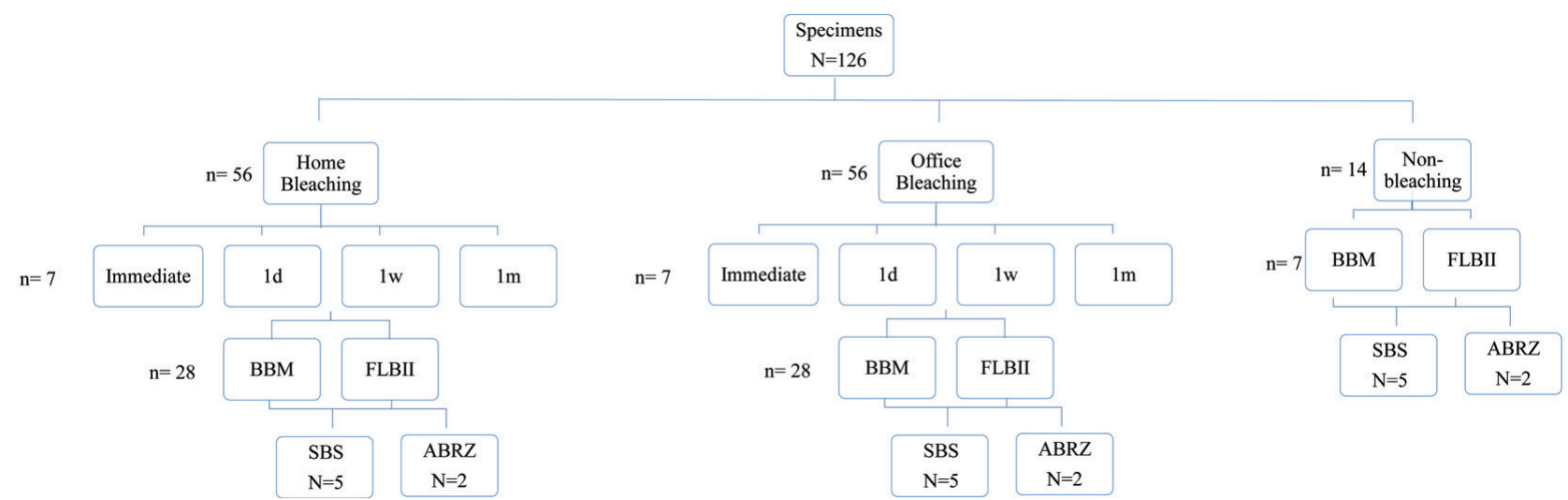

Fig. 2 Flow chart of specimen grouping.

groups were further divided randomly into 4 equal-sized subgroups according to storage period after completion of the bleaching process, namely, immediate, $1 \mathrm{~d}, 1 \mathrm{w}$ and $1 \mathrm{~m}$. Specimens were stored in distilled water at $37^{\circ} \mathrm{C}$ before the adhesive was applied. Each subgroup based on time was treated with either the one-step self-etch or two-step SEAs ( $n=28 /$ group) (Fig. 2).

In-office bleaching was performed on 56 specimens with Hi-Lite bleaching agent. One scoop of powder and three drops of liquid were mixed for $30 \mathrm{~s}$ using a spatula until a uniform paste was formed. The paste was applied 1-2 mm thick on the enamel surface of the specimens. The paste was left on the surface for $6 \mathrm{~min}$ and then irradiated with a quartz-halogen light curing unit (Optilux 501, Demetron, Danbury, CT, USA) with a power density of $550 \mathrm{~mW} / \mathrm{cm}^{2}$ for $3 \mathrm{~min}$. The paste was left on the surface for another $2 \mathrm{~min}$ and then removed with a damp cotton roll. This procedure was repeated three times, and the specimen was then rinsed under water and dried gently. After bleaching, the teeth were stored in distilled water at $37^{\circ} \mathrm{C}$ for the different times $1 \mathrm{~d}, 1 \mathrm{w}$ and $1 \mathrm{~m}$.

At-home bleaching was performed on 56 specimens with Hi-Lite shade up bleaching. The bleaching material was applied to the enamel surfaces with a brush and left for $2 \mathrm{~h}$. The procedure was repeated over 14 consecutive days, as recommended by the manufacturer. The specimens were stored in distilled water at $37^{\circ} \mathrm{C}$ between bleaching cycles. The bleaching agent was changed every day after the bleaching cycle was completed and stored in distilled water at different times $1 \mathrm{~d}, 1 \mathrm{w}$ and $1 \mathrm{~m}$.

\section{$\mu$ SBS testing}

After bonding resin application, tygon tubes (R-3603, Norton Performance Plastic, Cleveland, OH, USA), internal diameter of $0.79 \mathrm{~mm}$ and height of $0.5 \mathrm{~mm}(n=5$ teeth, each tooth bonded with 9 tubes), were placed on the bleached enamel surfaces and light-cured for $10 \mathrm{~s}$. A resin composite (Beautifil II, shade A2, Shofu Dental) was inserted into the tygon tubes and light polymerized for $40 \mathrm{~s}$ using the quartz-halogen light curing unit. After polymerization of the resin composite, the specimens were stored in distilled water at $37^{\circ} \mathrm{C}$ for 1 -day.

After the storage for 1-day in water, the tygon tubes were removed using a scalpel blade. Each specimen was attached to a jig (Bencor- Multi-T, Danville Engineering, San Ramon, CA, USA) using a cyanoacrylate glue (Model Repair, Dentsply-Sankin, Ohtawara, Japan) and placed in a universal testing machine (EZ-Test-500N, Shimadzu, Kyoto, Japan) to measure the $\mu$ SBS. A thin wire, with a $0.25 \mathrm{~mm}$ cross-section, was looped around the base of the resin composite cylinder bonded to the enamel surface and connected to a metal rod of the bond testing jig on the universal testing machine. A shear stress was applied at a crosshead speed of $1.0 \mathrm{~mm} / \mathrm{min}$ until bond failure occurred ${ }^{18)}$. 


\section{SEM observation}

1. Failure mode analysis

After the $\mu$ SBS testing, debonded areas of all specimens were gold sputter-coated to evaluate the failure patterns on the enamel surface using SEM (JSM- IT100LV, JEOL, Tokyo, Japan) at $\times 200$ magnification. Failure modes were classified into three types: Type A: adhesive failure at the interface between the bonding resin and enamel; Type B: cohesive failure in the bonding resin; and Type M: mixed failure including adhesive failure and cohesive failure in the bonding resins.

\section{ABRZ}

Thirty-six specimens were used for ABRZ observation in each experimental group $(n=2)$ as illustrated in Fig. 3. The specimens were prepared in the same manner as for the bond test. Additionally, a thin coating of resin composite (Clearfil AP-X, shade A2, Kuraray Noritake Dental, Tokyo, Japan) was applied on top of the bonded specimens and cured for $40 \mathrm{~s}$. After storage in distilled water at $37^{\circ} \mathrm{C}$ for 1 -day, each specimen was sectioned into two halves perpendicular to the bonded interface then embedded in slow-curing epoxy resin (Epoxicure Resin, Buehler) overnight. Specimens were wet-polished with $\mathrm{SiC}$ paper to 1200 -grit to standardize the surface and then subjected to an acid-base challenge with a demineralizing solution ( $\mathrm{pH} 4.5)$ for $2 \mathrm{~h}$ and $5 \% \mathrm{NaClO}$ for $20 \mathrm{~min}^{10)}$. A self-cure resin, Super-Bond C\&B (Sun Medical, Moriyama, Japan) was then applied to the treated surface without acid etching to prevent wear of the interface ${ }^{19)}$. The specimens were then cut perpendicular to the interface, polished with $\mathrm{SiC}$ paper to 2000-grit, and finished with diamond pastes to $0.25 \mu \mathrm{m}$ followed by argon-ion beam etching (EIS-IE, Elionix, Tokyo, Japan) of the polished surfaces to bring the adhesive/enamel interface into sharp relief, with an accelerating voltage of $1 \mathrm{kV}$ and an ion current density of $1.5 \mathrm{~mA} / \mathrm{cm}^{2}$ for $30 \mathrm{~s}$ in order to distinguish the different zones of the interface that were observed using SEM (JSM- IT100LV, JEOL) at $\times 3,500$ magnification.

\section{Statistical analysis}

Data were analyzed for normal distribution using the
Kolmogorov-Smirnov and Shapiro-Wilk tests. $\mu$ SBS data were analysed using three-way ANOVA followed by pairwise comparison with Bonferroni correction (IBM Released 2015. IBM SPSS Statistics for Windows, Version 23.0. IBM, Armonk, NY, USA) $(\alpha=0.05)$.

\section{RESULTS}

\section{$\mu S B S$}

The $\mu$ SBS values are listed in Table 2. Three-way ANOVA showed that "bleaching" did not have a significant effect ( $p=0.221)$, while, "adhesive" and "postbleaching period" showed significant effects on the $\mu$ SBS $(p \leq 0.001)$. The interaction between the 3 variables showed a significant effect $(p=0.018)$.

The $\mu \mathrm{SBS}$ of BBM was $16.6 \mathrm{MPa}$ in the control groups which was significantly lower than that of FLB II 27.6 $\mathrm{MPa}(p<0.01)$. The $\mu \mathrm{SBS}$ of in-office bleaching, for BBM, significantly decreased immediately after bleaching $(p<0.001)$ and increased 1d after bleaching. The bond strength recovered to the original value $1 \mathrm{w}$ and $1 \mathrm{~m}$ after bleaching. For FLB II, the $\mu$ SBS was significantly lower in the immediate group and increased slightly $1 \mathrm{~d}$ after bleaching, and recovered $1 \mathrm{w}$ after bleaching but did not achieve the same strength as the original value (27.6 $\mathrm{MPa}) 1 \mathrm{~m}$ after bleaching.

The $\mu$ SBS of in-office bleaching, in both adhesive materials showed a significant decrease in immediate groups and slightly increased after $1 \mathrm{~d}$ and had recovered by $1 \mathrm{w}$ and $1 \mathrm{~m}$ after bleaching. For the at-home bleaching, both adhesive materials showed a significant decrease in the immediate groups and slightly increased after $1 \mathrm{~d}$ and remained stable for BBM and FLBII but did not achieve the same bond strength as in the control groups for FLBII.

The $\mu \mathrm{SBS}$ of at-home bleaching, for BBM, significantly decreased immediately after bleaching $(p<0.001)$. However, the $\mu$ SBS recovered to the original value by $1 \mathrm{~d}, 1 \mathrm{w}$ and $1 \mathrm{~m}$ after bleaching. For FLB II, the $\mu$ SBS significantly decreased in the immediately after bleaching group $(p<0.001)$ which was not significantly different from that of FLB II after $1 \mathrm{~d}, 1 \mathrm{w}$ and $1 \mathrm{~m}$ of bleaching.
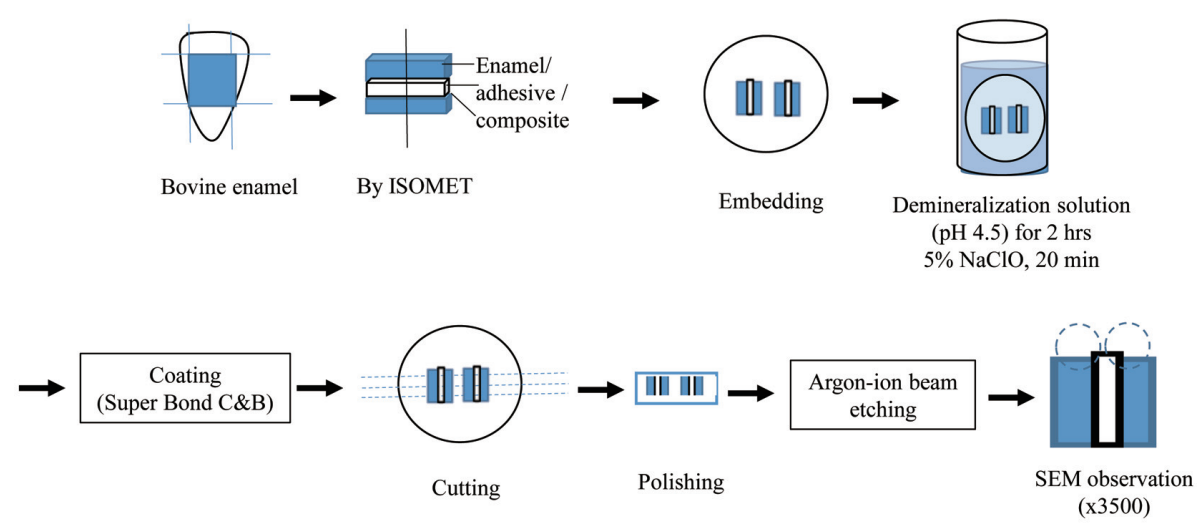

Fig. 3 Methodology of specimen preparation for SEM observation of ABRZ. 
Table 2 Effect of time after bleaching of in-office bleaching and at-home bleaching on $\mu$ SBS to enamel

\begin{tabular}{|c|c|c|c|c|}
\hline & Time & $\mathrm{BBM}$ & FLBII & $p$-value \\
\hline Control & - & $16.6 \pm 3.8^{\mathrm{ab}}$ & $27.6 \pm 5.0^{\mathrm{a}}$ & $\leq 0.001^{*}$ \\
\hline \multirow{4}{*}{ In-office bleaching } & Immediate & $4.5 \pm 1.9^{\mathrm{e}}$ & $6.6 \pm 4.0^{\mathrm{e}}$ & $0.096 \mathrm{NS}$ \\
\hline & $1 d$ & $12.7 \pm 3.3^{\mathrm{d}}$ & $19.8 \pm 4.4^{\mathrm{bc}}$ & $\leq 0.001^{*}$ \\
\hline & $1 \mathrm{w}$ & $18.0 \pm 5.3^{\mathrm{a}}$ & $25.7 \pm 5.1^{\mathrm{a}}$ & $\leq 0.001^{*}$ \\
\hline & $1 \mathrm{~m}$ & $16.3 \pm 4.7^{\mathrm{abc}}$ & $22.0 \pm 4.6^{\mathrm{b}}$ & $\leq 0.001^{*}$ \\
\hline \multirow{4}{*}{ At-home bleaching } & Immediate & $13.7 \pm 2.9^{\text {cd }}$ & $16.3 \pm 3.1^{\mathrm{d}}$ & $\leq 0.001^{*}$ \\
\hline & $1 d$ & $14.8 \pm 4.2^{\text {bcd }}$ & $19.7 \pm 4.9^{\mathrm{bc}}$ & $\leq 0.001^{*}$ \\
\hline & $1 \mathrm{w}$ & $15.6 \pm 4.2^{\mathrm{abcd}}$ & $17.7 \pm 5.6^{\mathrm{cd}}$ & $0.059 \mathrm{NS}$ \\
\hline & $1 \mathrm{~m}$ & $15.0 \pm 4.8^{\mathrm{bcd}}$ & $16.7 \pm 3.8^{\mathrm{cd}}$ & $0.131 \mathrm{NS}$ \\
\hline
\end{tabular}

Mean \pm Standard deviation with the same letters ( $\mathrm{a}, \mathrm{b}, \mathrm{c}$ or $\mathrm{d}$ ) within each column indicate no significant difference (NS) at $(p>0.001) . *$ significant difference.

Number of specimens: 45 for each group.

Table 3 Fracture mode analysis of debonded specimens after $\mu$ SBS test

\begin{tabular}{|c|c|c|c|}
\hline & Bleaching & $\mathrm{BBM} \%$ & FLBII \% \\
\hline Control & - & $36 / 0 / 9$ & $38 / 2 / 5$ \\
\hline \multirow{4}{*}{ In-office bleaching } & Immediate & $29 / 3 / 13$ & $32 / 4 / 9$ \\
\hline & $1 d$ & $22 / 3 / 20$ & $31 / 6 / 8$ \\
\hline & $1 \mathrm{w}$ & $28 / 0 / 17$ & 29/4/12 \\
\hline & $1 \mathrm{~m}$ & $16 / 2 / 17$ & $25 / 6 / 14$ \\
\hline \multirow{4}{*}{ At-home bleaching } & Immediate & $33 / 0 / 12$ & $41 / 0 / 4$ \\
\hline & $1 d$ & $30 / 3 / 12$ & $40 / 0 / 5$ \\
\hline & $1 \mathrm{w}$ & $34 / 0 / 11$ & $36 / 2 / 7$ \\
\hline & $1 \mathrm{~m}$ & $30 / 7 / 8$ & 18/8/19 \\
\hline
\end{tabular}

Type A; adhesive failure at the interface between the bonding resin and enamel, Type B; cohesive failure in the bonding resin, Type M; mixed failure including adhesive failure and cohesive failure in the bonding resin

\section{Failure mode analysis}

The failure mode results are shown in Table 3.

In the control group, for both BBM and FLB II, Type A (adhesive between enamel and resin) was the main failure mode, however, Type B (cohesive failure in bonding resin) was observed in FLB II in approximately $5 \%$ of specimens.

In-office bleaching, for BBM, Type B cohesive failure in the bonding resin were observed in all groups except at $1 \mathrm{w}$ which showed $60 \%$ adhesive failures (Type A) and 40\% mixed failures (Type M). For FLB II, 8-13\% Type $\mathrm{B}$ cohesive failures in bonding resin were observed in all groups.

At-home bleaching, for BBM, the main failure mode was Type A in all groups however, 7\% Type B (cohesive failure in bonding resin) was only observed at 1d. For FLB II, the main failure mode was Type A in all groups except for $1 \mathrm{~m}$, which exhibited $40 \%$ for mixed failure. Type B was observed in the $1 \mathrm{w}$ and $1 \mathrm{~m}$ groups.

The $\mu$ SBS of in-office and at-home bleaching, for both adhesive materials showed the main failure was adhesive (Type A). Mixed failure (Type M) increased at $1 \mathrm{~m}$ for FLB II and at $1 \mathrm{~d}, 1 \mathrm{w}$ and $1 \mathrm{~m}$ for in-office bleaching with BBM.

\section{$A B R Z$}

Typical SEM images of the enamel-adhesive interface for each group after acid-base challenge are shown in Figs. 4 (control), 5 (in-office bleaching), and 6 (at-home bleaching), respectively. The outer lesion (OL), created by dissolution of enamel due to the acid-base challenge was observed in each group and the depth of the OL ranged from 10 to $15 \mu \mathrm{m}$. ABRZ was observed at the demineralization front of OL in all groups.

In the control groups, for BBM, the ABRZ was 0.5 $\mu \mathrm{m}$ thick (Fig. 4a). A funnel-shaped lesion (lightening symbol) formed at the base of the OL in BBM (Fig. 4a). In FLB II (Fig. 4b), the ABRZ was approximately $1 \mu \mathrm{m}$ thick, sloped and increased from the top up to the end of OL without a funnel-shaped lesion.

In office bleaching, in the BBM groups, the ABRZ increased to a thickness of about $3 \mu \mathrm{m}$ in the immediate and $1 \mathrm{~d}$ after bleaching groups and returned back to approximately $0.5 \mu \mathrm{m}$ thick with a funnel-shaped lesion in the $1 \mathrm{w}$ and $1 \mathrm{~m}$ after bleaching group (Figs. 5a-d). For the FLB II groups (Fig. 5e-h), the ABRZ increased to a 


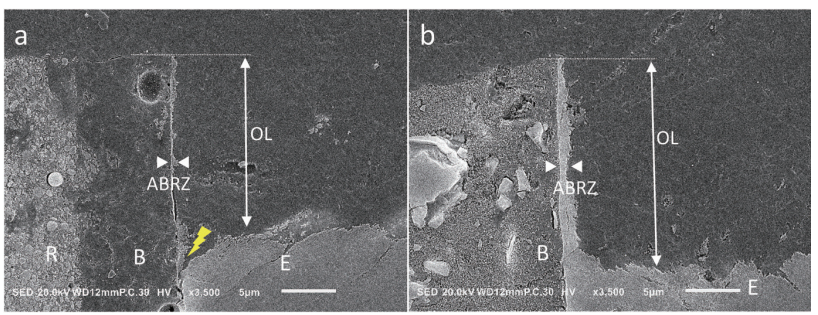

Fig. 4 SEM image of enamel-adhesive interface after acid- base challenge in the control group.

OL: outer lesion; B: bonding; E: Enamel; R: resin; ABRZ: acid-base resistant zone. a- BBM, and bFLBII. ABRZ was detectable in all groups. thickness of about $3 \mu \mathrm{m}$ in the immediate, $1 \mathrm{~d}, 1 \mathrm{w}$ and $1 \mathrm{~m}$ after bleaching groups.

For at-home bleaching, in the BBM groups, the ABRZ was $3 \mu \mathrm{m}$ thick in the immediate and $1 \mathrm{~d}$ groups with the presence of a funnel-shaped lesion in the immediate group. The $1 \mathrm{w}$ and $1 \mathrm{~m}$ after bleaching groups showed an ABRZ approximately 3 to $5 \mu \mathrm{m}$ thick, however, the funnel-shaped lesion could not be observed (Figs. 6a-d). For the FLB II groups, the ABRZ increased to a thickness of about 3 to $5 \mu \mathrm{m}$ in the immediate, $1 \mathrm{~d}, 1 \mathrm{w}$ and $1 \mathrm{~m}$ after bleaching groups (Figs. 6e-h).

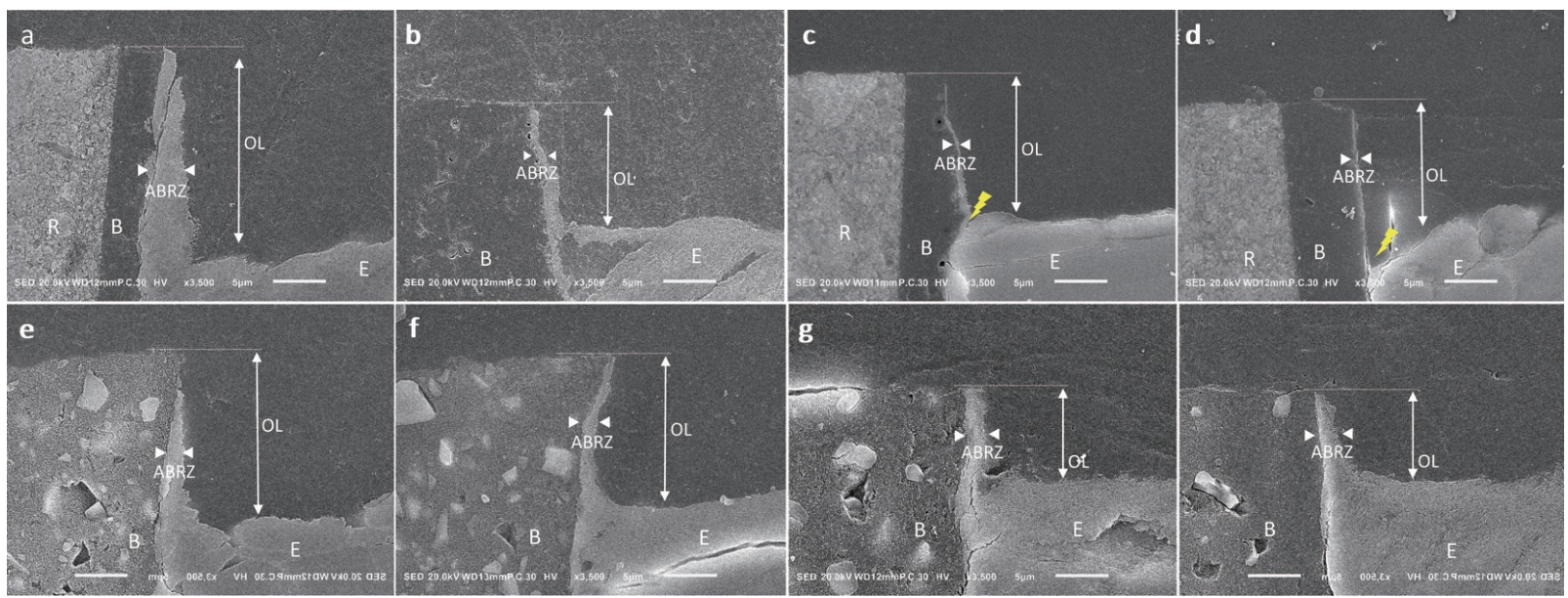

Fig. 5 SEM image of enamel-adhesive interface after acid-base challenge of in-office bleaching groups.

OL: outer lesion; B: bonding; E: Enamel; R: resin; ABRZ: acid-base resistant zone. a- BBM with bleaching immediate, b- BBM with bleaching one day, c- BBM with bleaching one week, d- BBM with bleaching one month, e- FLBII with bleaching immediate, f- FLBII with bleaching one day, g- FLBII with bleaching one week, and h- FLBII with bleaching one month. ABRZ was detectable in all groups.
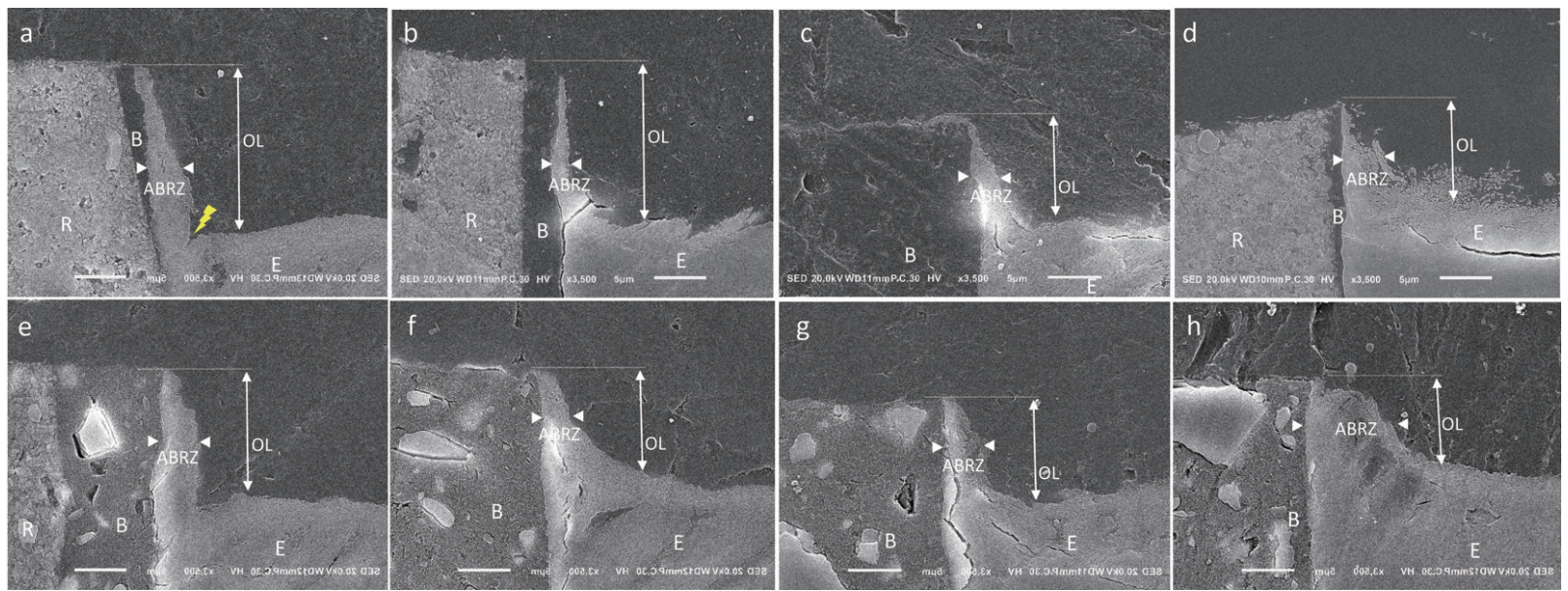

Fig. 6 SEM image of enamel-adhesive interface after acid-base challenge of at-home bleaching groups.

OL: outer lesion; B: bonding; E: Enamel; R: resin; ABRZ: acid-base resistant zone. a- BBM with bleaching immediate, b- BBM with bleaching one day, c- BBM with bleaching one week, d- BBM with bleaching one month, e- FLBII with bleaching immediate, f- FLBII with bleaching one day, g- FLBII with bleaching one week, and h- FLBII with bleaching one month. ABRZ was detectable in all groups. 


\section{DISCUSSION}

Many bleaching products and techniques are now available for professional and non- professional use. Patients might be scheduled for orthodontic or restorative treatment shortly after bleaching.

Bovine teeth resemble human teeth in many characteristics $^{20)}$ and have been used to compare the bond strength of bleached enamel with that of unbleached enamel ${ }^{21)}$. In order to simulate the clinical situation, an intact enamel surface should be used. But for better standardization and to understand the effect of the bleaching agent on bond strength and ABRZ enamel surfaces a flat surface were created.

For in-office bleaching, the $\mu \mathrm{SBSs}$ for the one-step and two-step SEA systems decreased immediately after bleaching followed by a gradual increase 1d after bleaching and completely recovered to bond strengths the same as the control group after $1 \mathrm{w}$. The immediate decrease in bond strength after bleaching may be due to the presence of residual oxygen from the bleaching agent, which inhibits resin polymerization. The delayed application of bonding by $1 \mathrm{w}$ seems to eliminate the effect of residual oxygen. Spyrides et al. ${ }^{22)}$, also reported decreased bond strengths when bleached teeth were immediately bonded, while, there was a significant increase in bond strength when bonding was delayed for a week. Other studies reported delaying bonding after bleaching for 2 to 4 weeks, to eliminate the detrimental effects of residual oxygen from the bleaching agent which inhibited resin polymerization and interfered with resin attachment ${ }^{22,23)}$.

For at-home bleaching, the $\mu$ SBS of BBM slightly decreased immediately after bleaching and returned to the original level by $1 \mathrm{w}$. On the other hand, for FLB II, the $\mu$ SBS decreased immediately after bleaching, and also $1 \mathrm{~d}$ and 1w. BBM is an acetone-solvated adhesive which might minimize the inhibitory effects of oxygen due to the interaction between acetone and oxygen. FLB II has multi-ion-releasing components, which might be the reason the $\mu$ SBS did not recover until $1 \mathrm{~m}$ after at-home bleaching, but this still remains unclear. One study reported that ethanol or acetone-based adhesive systems counteract the effect of whitening because of their water-clearing effect ${ }^{24)}$. Gungor et al. ${ }^{25)}$ reported that at-home bleaching affected the $\mu$ SBS more adversely. They explained that the longer application periods associated with the at-home bleaching method caused alterations in the organic substance in the enamel and the loss of calcium may also increase over time. Another study reported that the normal $\mu \mathrm{SBS}$ values attained in an at-home bleaching group were probably attributable to the lower concentration of the hydrogen peroxide (10\%) which was mainly because, after each daily bleaching, the teeth were stored in distilled water. This might have eliminated the residual peroxide absorbed by the enamel ${ }^{26)}$.

The majority of failures in all groups were adhesive (Type A). However, for at-home bleaching at $1 \mathrm{~m}$ there was an increase in cohesive failure in the bonding resin for both adhesive systems. The cohesive failures of the adhesive materials were often associated with high bond strength values, indicating effective bonding. In this case, an increase in cohesive failure of the bonding resin probably means lower mechanical properties of the adhesive possibly due to poorer polymerization.

The characteristics of the ABRZs for FLBII were thicker than that of BBM. A thicker ABRZ was found in the FLBII groups, because FLBII contains a multi-ionreleasing component and S-PRG filler in the adhesive. Fluoride release from adhesives was reported to be a key factor to create thick ABRZs ${ }^{10)}$. The ABRZ of FLBII sloped and increased from the top to the end of the OL, while the ABRZs for BBM were parallel and homogeneous. The slope formation of FLBII is believed to be due to fluoride-release from the adhesive. ABRZ formation may be due to the potential for monomer penetration and fluoride release in the adhesive systems ${ }^{10)}$. However, a funnel-shaped lesion adjacent to the ABRZ was observed in BBM, which is similar to that found in previous studies $^{9,12,14)}$, suggesting a weak area vulnerable to acidbase attack beneath the bonding interface.

On the other hand, when enamel was bleached using either the at-home or in-office bleaching, the thickness of the ABRZ was obviously increased and showed an irregular shape. This may be due to an increase in enamel porosity after bleaching which led to deeper penetration of the primer. A previous study suggested that bleaching enamel may increase enamel porosity, leading to deeper penetration of the self-etching primer, however, residual oxygen from the bleaching agent may hinder polymerization of the adhesive, thus causing a decrease in the $\mu \mathrm{SBS}^{12)}$. In a previous study, SEM examination confirmed that the application of a bleaching agent produced an aggressive etching pattern ${ }^{27)}$. The resin monomers were able to penetrate more deeply into the demineralized layer, resulting in thicker ABRZ formation. One study reported that the change of storage water may have has also contributed to mineral loss from dentin surfaces due to deionized water lacking calcium and phosphate ions and therefore possessing a higher chemical potential for dissolution of the mineral leaving unprotected collagen fibril ${ }^{28)}$. In the current study, the loss of mineral from the enamel was also reflected by a gradual increase in OL depth, which was significantly correlated with storage time. In-office bleaching showed an ABRZ thickness that decreased by $1 \mathrm{w}$ and $1 \mathrm{~m}$ in both adhesive and lesion formation for BBM. This was presumably because enamel porosity decreased, so primer penetration was not as deep as in the immediate and $1 \mathrm{~d}$ after bleaching groups which led to a thinner ABRZ.

\section{CONCLUSIONS}

Based on the results of this study, the following conclusions could be made.

In-office and at-home bleaching adversely affected the $\mu$ SBS to enamel when bonded immediately and after 1 day, however, the $\mu$ SBS values had recovered by 1 
week after cessation of the bleaching process.

Delaying bonding for at least 1 week after in-office or at-home bleaching is recommended.

Thick ABRZs were created with in-office bleaching and at-home bleaching for all storage times except for BBM which had a thin ABRZ at $1 \mathrm{w}$ and $1 \mathrm{~m}$ after inoffice bleaching application.

\section{CLINICAL RELEVANCE}

It is suggested that the present study regimen be carried out under clinical conditions and both adhesive systems undergo delayed bonding at different intervals so that the minimum delay in bonding can be determined for the two bonding systems.

\section{ACKNOWLEDGMENTS}

This study was partly supported by a grant from the Japan Society for the Promotion of Science (16H05515).

\section{REFERENCES}

1) Bishara SE, Oonsombat C, Soliman MMA, Ajlouni R, Laffoon JF. The effect of tooth bleaching on the shear bond strength of orthodontic brackets. Am J Orthod Dentofacial Orthop 2005; 128: 755-760.

2) Basting R, Amaral F, França F, Flório F. Clinical comparative study of the effectiveness of and tooth sensitivity to $10 \%$ and $20 \%$ carbamide peroxide home-use and $35 \%$ and $38 \%$ hydrogen peroxide in-office bleaching materials containing desensitizing agents. Oper Dent 2012; 37: 464-473.

3) Kavitha M, Selvaraj S, Khetarpal A, Raj A, Pasupathy S, Shekar S. Comparative evaluation of superoxide dismutase, alpha-tocopherol, and 10\% sodium ascorbate on reversal of shear bond strength of bleached enamel: An in vitro study. Eur J Dent 2016; 10: 109-115.

4) Yoshiyama M, Doi J, Nishitani Y, Itota T, Tay FR, Carvalho $\mathrm{RM}$, et al. Bonding ability of adhesive resins to caries-affected and caries-infected dentin. J Appl Oral Sci 2004; 12: 171176.

5) Han L, Edward C, Okamoto A, Iwaku M. A comparative study of fluoride-releasing adhesive resin materials. Dent Mater J 2002; 21: 9-19

6) Torii $Y$, Itota $\mathrm{T}$, Okamoto $\mathrm{M}$, Nakabo $\mathrm{S}$, Nagamine $\mathrm{M}$, Inoue $\mathrm{K}$. Inhibition of artificial secondary caries in root by fluoridereleasing restorative materials. Oper Dent 2001; 26: 36-43.

7) Preston AJ, Mair LH, Agalamanyi EA, Higham SM. Fluoride release from aesthetic dental materials. J Oral Rehabil 1999; 26: 123-129.

8) Tsuchiya S, Nikaido T, Sonoda H, Foxton RM, Tagami J. Ultrastructure of the dentin-adhesive interface after acidbase challenge. J Adhes Dent 2004; 6: 183-190.

9) Sato T, Takagaki T, Matsui N, Hamba H, Sadr A, Nikaido $\mathrm{T}$, et al. Morphological evaluation of the adhesive/enamel interfaces of two-step self-etching adhesives and multimode one-bottle self-etching adhesives. J Adhes Dent 2016; 18: $1-5$.

10) Li N, Nikaido T, Takagaki T, Sadr A, Makishi P, Chen J, et al. The role of functional monomers in bonding to enamel: Acidbase resistant zone and bonding performance. J Dent 2010; 38: 722-730.

11) Li N, Nikaido T, Alireza S, Takagaki T, Chen JH, Tagami J. Phosphoric acid-etching promotes bond strength and formation of acid-base resistant zone on enamel. Oper Dent
2013; 38: 82-90.

12) Halabi $S$, Matsui N, Nikaido T, Burrow MF, Tagami J. Effect of office bleaching on enamel bonding performance. J Adhes Dent 2019; 21: 167-177.

13) Guan R, Takagaki T, Matsui N, Sato T, Burrow Mf, Palamara $\mathrm{J}$, et al. Dentin bonding performance using Weibull statistics and evaluation of acid-base resistant zone formation of recently introduced adhesives. Dent Mater J 2016; 35: 684693.

14) Nikaido T, Ichikawa C, Li N, Takagaki T, Sadr A, Yoshida $\mathrm{Y}$, et al. Effect of functional monomers in all-in-one adhesive systems on formation of enamel/dentin acid-base resistant zone. Dent Mater J 2011; 30: 576-582.

15) Shinohara MS, Yamauti M, Inoue G, Nikaido T, Tagami J, Giannini M, et al. Evaluation of antibacterial and fluoridereleasing adhesive system on dentin-Microtensile bond strength and acid-base challenge. Dent Mater J 2006; 25: 545-552.

16) Iida $Y$, Nikaido T, Kitayama S, Takagaki T, Ikeda M, Foxton $\mathrm{RM}$, et al. Evaluation of dentin bonding performance and acid-base resistance of the interface of two-step self-etching adhesive systems. Dent Mater J 2009; 28: 493-500.

17) Abed Kahnemooyi M, Ajami AA, Kimai S, Fatemeh Pournaghiazar, Siavash Oskoee S, Mhammadi Torkani MA. Effect of sodium ascorbate and delayed bonding on the bond strength of silorane and two-step self-etch adhesive systems in bleached enamel. J Dent Res Dent Clin Dent Prospect 2014; 8: 210-217.

18) Shimada Y, Iwamoto N, Kawashima M, Burrow MF, Tagami J. Shear bond strength of current adhesive systems to enamel, dentin and dentin-enamel junction region. Oper Dent 2019; 28: 585-590.

19) Inoue G, Tsuchiya S, Nikaido T, Foxton RM, Tagami J. Morphological and mechanical characterization of the acidbase resistant zone at the adhesive-dentin interface of intact and caries-affected dentin. Oper Dent 2006; 31: 466-472.

20) Sayed M, Matsui N, Hiraishi N, Nikaido T, Burrow M, Tagami J. Effect of glutathione bio-molecule on tooth discoloration associated with silver diammine fluoride. Int J Mol Sci 2018; 19: 1322 .

21) Miles PG, Pontier JP, Bahiraei D, Close J. The effect of carbamide peroxide bleach on the tensile bond strength of ceramic brackets: An in vitro study. Am J Orthod Dentofacial Orthop 1994; 106: 371-375.

22) Spyrides GM, Perdigão J, Pagani C, Araújo MA, Spyrides SM. Effect of whitening agents on dentin bonding. J Esthet Dent 2000; 12: 264-270.

23) Titley KC, Torneck CD, Ruse ND. The effect of carbamideperoxide gel on the shear bond strength of a microfil resin to bovine enamel. J Dent Res 1992; 71: 20-24.

24) Yazici AR, Keleş A, Tuncer D, Başeren M. Effect of prerestorative home-bleaching on microleakage of self-etch adhesives. J Esthet Restor Dent 2010; 22: 186-192.

25) Gungor AY, Ozcan E, Alkis H, Turkkahraman H. Effects of different bleaching methods on shear bond strengths of orthodontic brackets. Angle Orthod 2013; 83: 686-690.

26) Patusco VC, Montenegro G, Lenza MA, De Carvalho AA. Bond strength of metallic brackets after dental bleaching. Angle Orthod 2009; 79: 122-126.

27) Pimenta-Dutra AC, Albuquerque R de C, Morgan LF dos SA, Pereira GM, Nunes E, Horta MCR, et al. Effect of bleaching agents on enamel surface of bovine teeth: A SEM study. J Clin Exp Dent 2017; 9: 46-50.

28) Ichikawa C, Nikaido T, Inoue G, Sadr A, Tagami J. Ultramorphological evaluation of the dentin acid-base resistant zone of two-step self-etching systems after longterm storage in water. J Adhes Dent 2012; 14: 207-213. 